\title{
The pointedness effect on representational momentum
}

\author{
MASAYOSHI NAGAI and AKIHIRO YAGI \\ Kwansei Gakuin University, Hyogo, Japan
}

\begin{abstract}
An observer's memory for the final position of a moving object is shifted forward in the direction of that object's motion. It is called representational momentum (RM). This study addressed stimulusspecific effects on RM. In Experiment 1, participants showed larger memory shift for an object moving in its typical direction of motion than when it moved in a nontypical direction of motion. In Experiment 2, participants indicated larger memory shift for a pointed pattern moving in the direction of its point than when it moved in the opposite direction. In Experiment 3, we again examined the influences of knowledge about objects' typical motions and the pointedness of objects, because we did not control the shape (pointedness) of objects in Experiment 1. The results showed that only pointedness affected the magnitude of memory shift and that the effect was smaller than the momentum effect.
\end{abstract}

When motion of a stimulus is implied, an observer's memory for the final position of the stimulus is shifted forward in the direction of the stimulus's motion. Freyd and Finke (1984) termed this forward memory shift representational momentum (RM), because it is similar to physical momentum. A physical object in motion cannot stop immediately but coasts some distance because of its momentum. Similarly, an observer's mental representation of an object in motion acquires a momentum-like characteristic and cannot stop immediately. This analogy suggests that RM occurs because the law of physical momentum has been incorporated into the representational system, as a consequence of possible selective or survival advantages (Hubbard, 1998, 1999; Kelly \& Freyd, 1987).

RM and other memory shifts reflect physical principles. Forward memory shift increases with the velocity of a stimulus (Freyd \& Finke, 1985; Hubbard \& Bharucha, 1988) and acceleration (Finke, Freyd, \& Shyi, 1986). Downward motion of a stimulus produces larger forward memory shift than does upward motion (representational gravity; Hubbard, 1990; Hubbard \& Bharucha, 1988) and implied friction between a moving stimulus and an adjoining surface reduces forward memory shift (representational friction; Hubbard, 1995a).

The direction and the magnitude of memory shift were also influenced by observers' expectations regarding the future behavior of a stimulus (expectation effect; Hub-

We express appreciation to Koji Kazai, Nobuyuki Kawai, Akihiko Iwahara, and Yuji Takeda for their advice on this project and to Hiroko Fukuda, Gregory G. Anderson, and Frank Thomas for improving the English expression of this article. We also thank David E. Irwin, Timothy P. McNamara, Timothy L. Hubbard, and an anonymous reviewer for their helpful comments on an earlier draft of this manuscript. Correspondence concerning this article should be addressed to M. Nagai, Graduate School of Informatics, Kyoto University, Yoshida Honmachi, Sakyo-ku, Kyoto, 606-8501, Japan (e-mail: nagai@ cog.ist.i.kyoto-u.ac.jp). bard \& Bharucha, 1988; Verfaillie \& d'Ydewalle, 1991). Hubbard and Bharucha presented a circle stimulus moving horizontally within a frame. The circle bounced off the inside of the frame from one to five times, and both the circle and the frame vanished simultaneously. Memory shift was observed in the direction of the circle's motion when the circle vanished immediately after it collided with the frame, but memory shift was observed in the opposite (or expected) direction of the circle's motion when it vanished just before the collision. Verfaillie and d'Ydewalle presented a rectangle rotating back and forth periodically. They compared the magnitude of forward memory shift observed when the rectangle vanished at the boundary of the periodic motion with that observed when it vanished at the midpoint of the periodic motion. The magnitude of forward memory shift was greatly reduced when the rectangle vanished at the boundary, but standard forward memory shift was observed when it vanished at the midpoint.

In most RM studies, simple patterns (e.g., a circle or a rectangle) were used as stimuli, but several studies used familiar objects from the real world. Pictures of a fox, a motorcycle, a rhinoceros, a truck, and a ball were used as stimuli in Halpern and Kelly's (1993) study, which showed mainly that a stimulus moving rightward produced larger forward memory shift than did a stimulus moving leftward (rightward bias in RM). If observers' knowledge about the difference in typical velocity between these objects influenced the magnitude of memory shift, the motorcycle and the truck would produce larger forward memory shift than the others. However, they could not find any influence of object type, except that the truck produced larger forward memory shift than did the others in their first experiment. In contrast, Reed and Vinson (1996) showed that observers' knowledge about the typical directions of objects' motions affected the magnitude of memory shift when the shape (pointedness) of the pictures of objects presented was kept constant. In one of 
their experiments, forward memory shift for an object's ascending motion was greater when that object was a rocket than when it was a church steeple. In another experiment, the same pointed pattern was presented for two different groups. One group was told that the object was a rocket, and the other group was told that it was a church steeple. Those told that it was a rocket showed greater forward memory shift for its ascending motion than did the other. In summary, the findings concerning the influences of knowledge about objects' typical motions on RM was not consistent between these two studies. In other words, although a typical-velocity effect was not observed (Halpern \& Kelly, 1993), a typical-direction effect was found (Reed \& Vinson, 1996).

There might be another stimulus-specific RM effect. Freyd and Pantzer (1995) showed that picture of an arrow moving forward produced larger forward memory shift than did a picture of an arrow moving backward. Freyd and Miller (1992) found that a picture of a creature (a bird) moving forward yielded larger forward memory shift than did a picture of a creature moving backward. These findings might be another piece of evidence for a typical-direction effect, because one knows that an arrow and a bird typically move forward. However, the results might imply that a pointed-shape object moving in the direction of its point produced larger forward memory shift than did a pointed-shape object moving in the opposite direction, because the objects used in these studies had a clear sharp point at their fronts.

It was unclear whether the findings of Freyd and Miller (1992) and Freyd and Pantzer (1995) were due to a typical-direction effect or to a pointedness effect. Thus, the influence of objects' typical motions on RM was demonstrated in only one study (Reed \& Vinson, 1996). The effect of the pointedness of objects on RM was not investigated directly. The purpose of the present study was to reexamine the typical-motion effect and to investigate the pointedness effect empirically. In an original RM study (Freyd \& Finke, 1984), implied or discrete motion was used. In all the present experiments, however, observers saw pictures of objects moving smoothly, because we assumed that more natural or real presentation of objects' motions would fully evoke observers' knowledge about the objects' typical motions.

\section{EXPERIMENT 1}

In Experiment 1, we examined whether or not knowledge about objects' typical motions (typical directions and typical velocities) influenced RM. In each trial, the observers saw a picture of an airplane or a car moving forward or backward. It is common knowledge that in the real world, an airplane flies only forward ${ }^{1}$ and a car runs primarily forward but sometimes backward. If RM is affected by such knowledge about typical directions of objects' motions, we could expect that the airplane's moving forward would produce larger forward memory shift than the airplane's moving backward. As for the car, however, there would be no difference or a smaller difference in the magnitude of forward memory shift between forward and backward motions.

However, the same results would also be predicted for the influence of knowledge about the difference in typical velocity between the object's forward and backward motions. The airplane flies fast in its forward movement but cannot fly backward (its typical velocity is zero). The car runs faster in its forward movement than in its backward movement. This knowledge about such typical velocities would lead to the same prediction as the knowledge about typical directions.

We also examined the rightward bias in RM found by Halpern and Kelly (1993).

\section{Method}

Participants. Fourteen undergraduate students at Kwansei Gakuin University volunteered to participate in the present experiment. All the participants had normal or corrected-to-normal vision. They were naive as to the hypothesis.

Apparatus. The stimuli were presented on a 17-in. CRT monitor (MAG, MX17S) controlled with a computer (Apple Macintosh, Quadra 840AV). The monitor, with a refresh rate of $75 \mathrm{~Hz}$ and a resolution of $832 \times 624$ pixels, was placed at a distance of $80 \mathrm{~cm}$ from the eyes of the participants. The signals of their responses were supplied to the computer with a Key Pad (Advanced Gravis, Mac Gamepad). The resolution of a reaction time (RT) was $16 \mathrm{msec}$.

Stimuli. We used pictures of objects as stimuli: pictures of a leftfacing and a right-facing airplane and of a left-facing and a rightfacing car (Figure 1A). The pictures were drawn with 11 grays of 256 colors on a white background and were 1.9û (76 pixels) long.

Each trial consisted of a sequence of seven frames. Each frame contained the same picture of an object in various locations. The first six frames formed a motion display, and the seventh frame was a probe. The picture first appeared at $3.75^{\circ}$ (150 pixels) left or right of the center of the screen, and each picture in the next five frames was displaced horizontally by $0.75^{\circ}$ (30 pixels) toward the center of the screen. The duration of each frame was about $83 \mathrm{msec}$, and the interstimulus interval (ISI) was $0 \mathrm{msec}$. The picture in the sixth frame was always presented at the center of the screen. The speed of the motion was about $7.5 \mathrm{deg} / \mathrm{sec}$ (300 pixels/sec), and the duration of the motion was about $500 \mathrm{msec}$. In the motion display, the participants perceived a leftward or rightward horizontal apparent motion of the picture. The ISI between the sixth frame and a probe (the retention interval) was about $250 \mathrm{msec}$. A probe was presented at the same position as the sixth frame picture or was displaced horizontally by $-0.500^{\circ},-0.375^{\circ},-0.250^{\circ},-0.125^{\circ},+0.125^{\circ},+0.250^{\circ}$, $+0.375^{\circ}$, or $+0.500^{\circ}(-20,-15,-10,-5,+5,+10,+15$, or +20 pixels). The "+" sign indicated a displacement in the direction of the picture's motion, and the "-" sign indicated a displacement in the opposite direction. The probe remained on the screen until the participant responded. The intertrial interval was about $2 \mathrm{sec}$.

Procedure. Each participant sat on a chair in a dark room. The task of the participants was to press one of two buttons if the probe was presented at the same position as the picture in the last frame of the motion display and the other button if it was presented at different positions. The participants were instructed to respond as quickly as possible but to maintain a high degree of accuracy.

There were eight conditions: the AFL, AFR, ABL, ABR, CFL, CFR, CBL, and CBR conditions. The letters " $A$ " and " $C$ " indicate the airplane and the car, respectively. The letters " $F$ " and " $B$ " indicate the object's forward and backward motions, respectively. The letters "L" and "R" indicate the object's leftward and rightward motions, respectively. For example, in the AFL (airplane, forward and 
A
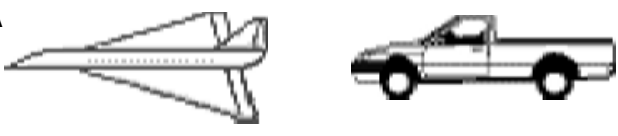

B
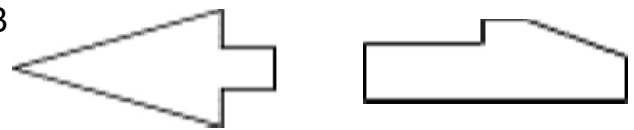

C
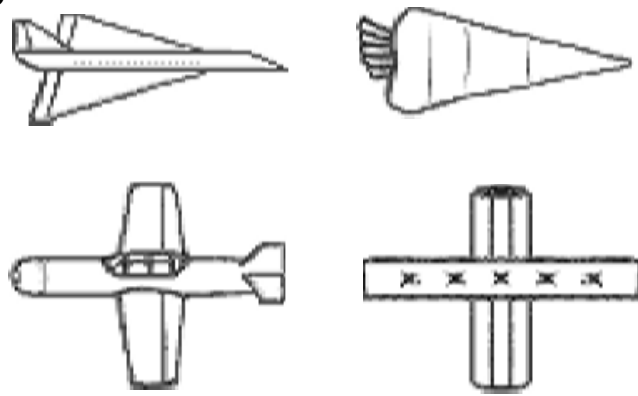

Figure 1. Stimuli used in Experiments 1-3. (A) Pictures of an airplane and a car used in Experiment 1. (B) Pictures similar to the outlines of the objects used in Experiment 1. These pictures were used in Experiment 2. (C) Pictures of a triangle-shaped airplane, a triangle-shaped carrot, a cross-shaped airplane, and a cross-shaped signpost, used in Experiment 3. The pictures of the airplane used in Experiment 1 were very similar to the pictures of the triangle-shaped airplane used in Experiment 3, but their lengths differed slightly (see the text). These pictures were drawn with 11 grays of 256 colors on a white background. It should be noted that, in the present study, left-facing and right-facing pictures of each object were used.

leftward) condition, the picture of the left-facing airplane moved leftward in the motion display, and in the ABR (airplane, backward and rightward) condition, the same picture (the left-facing airplane) moved rightward. The participants saw the airplane's forward motion from the right to the center of the screen in the AFL condition and the airplane's backward motion from the left to the center of the screen in the ABR condition. ${ }^{2}$
Each participant received 360 trials: 2 (objects: airplane or car) $\times 2$ (moving forward or backward) $\times 2$ (moving leftward or rightward) $\times 9$ displacements of probes $\times 5$ replications. Each participant received these trials in a different random order. All the trials were preceded by 30 practice trials that sampled all the displacements of probes and all the types of objects. No error feedback was given throughout the entire session. Responses and RT data were recorded. The entire session lasted about $70 \mathrm{~min}$.

To eliminate trials in which the participants lost their attention or pressed the response button inattentively, the trials in which the RTs were less than $150 \mathrm{msec}$ or three $S D$ s away from the participant's mean were discarded.

\section{Results and Discussion}

The mean percentage of trials eliminated was $1.81 \%$ $(0.56 \%-3.06 \%)$. In order to estimate the magnitude of memory shift, we used the weighted mean (based on Faust, 1990). The weighted means for each participant for each condition were calculated by adding up the products of the proportion of the same responses for each probe and the displacement size of that probe $\left(-0.5^{\circ}\right.$ to $\left.+0.5^{\circ}\right)$ and dividing the sum of the products by the sum of the proportion of the same responses. A positive value indicates memory shift in the direction of an object's motion (RM, or forward memory shift), and a negative value reflects memory shift in the opposite direction of its motion (backward memory shift). Zero indicates no memory shift. Table 1 shows the proportions of the same responses for each probe and the weighted means for each condition.

The $t$ tests revealed that the weighted means for all the conditions were significantly larger than zero [all $t \mathrm{~s}(13)>$ 3.46 , all $p$ s <.01]. Thus, it was confirmed that RM (forward memory shift) occurred in all the conditions. The weighted means were analyzed in a 2 (object: airplane or car) $\times 2$ (FB motion: forward or backward) $\times 2$ (LR motion: leftward or rightward) analysis of variance (ANOVA). A significant main effect for FB motion was found $[F(1,13)=10.08, p<.01]$. The other main effects were not significant. An object $\times$ FB motion interaction $[F(1,13)=11.02, p<.01]$ was significant. However, the other interactions were not significant.

Typical-motion effect. The statistical concern focused on the significant object $\times$ FB motion interaction, which reflected the larger difference in the magnitude of for-

Table 1

Mean Proportions of the Same Responses for Each Probe and Mean Weighted Means for Each Condition in Experiment 1

\begin{tabular}{|c|c|c|c|c|c|c|c|c|c|c|}
\hline \multirow[b]{2}{*}{ Condition } & \multicolumn{9}{|c|}{ Displacement of Probe (deg) } & \multirow[b]{2}{*}{ Weighted Mean (deg) } \\
\hline & -0.50 & -0.38 & -0.25 & -0.13 & 0 & +0.13 & +0.25 & +0.38 & +0.50 & \\
\hline \multicolumn{11}{|l|}{ Airplane } \\
\hline AFL & .03 & .03 & .09 & .35 & .53 & .83 & .68 & .47 & .27 & .14 \\
\hline AFR & .01 & .06 & .10 & .29 & .58 & .74 & .68 & .45 & .39 & .18 \\
\hline $\mathrm{ABL}$ & .01 & .03 & .21 & .55 & .75 & .64 & .48 & .23 & .23 & .09 \\
\hline $\mathrm{ABR}$ & .06 & .03 & .19 & .28 & .73 & .83 & .64 & .49 & .21 & .12 \\
\hline \multicolumn{11}{|l|}{ Car } \\
\hline CFL & .01 & .06 & .07 & .48 & .78 & .84 & .61 & .33 & .19 & .11 \\
\hline CFR & .01 & .06 & .20 & .39 & .67 & .83 & .71 & .51 & .29 & .14 \\
\hline CBL & .03 & .03 & .20 & .37 & .72 & .81 & .65 & .23 & .14 & .09 \\
\hline CBR & .01 & .04 & .10 & .36 & .74 & .72 & .62 & .40 & .24 & .14 \\
\hline
\end{tabular}

Note-The "-" sign indicates a displacement of a probe in the opposite direction of motion of an object. The "+" sign indicates a displacement of a probe in the same direction of motion of an object. A, airplane; C, car; F, forward; B, backward; L, leftward; R, rightward. 


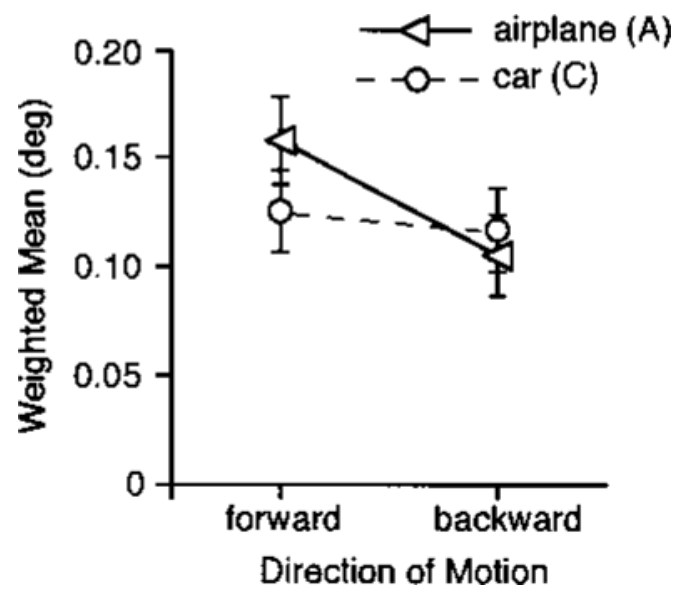

Figure 2. The weighted means for the airplane's forward (AF) and backward (AB) motions and for the car's forward $(\mathrm{CF})$ and backward (CB) motions in Experiment 1. These values arose from averaging the weighted means for the conditions over the leftward/rightward motions. Error bars indicate standard errors of the mean (SEMs).

ward memory shift between the airplane's forward (AF: $M=0.16$ ) and backward motions (AB: $M=0.11$ ) but a much smaller difference between the car's forward (CF: $M=0.12$ ) and backward motions (CB: $M=0.12$ ). This trend is shown in Figure 2. As to the significant object $X$ FB motion interaction, we performed post hoc Tukey's HSD tests $(p<.05)$. They revealed that AF produced larger forward memory shift than did $\mathrm{AB}, \mathrm{CF}$, and $\mathrm{CB}$.

As was predicted in the introduction to Experiment 1, the significant interaction suggested that RM was influenced by knowledge about objects' typical motions (typical directions and/or velocities of objects' motions). In addition, the significant difference in the weighted means between $\mathrm{AF}$ and $\mathrm{CF}$ might be due to knowledge about the difference in typical velocity between distinct objects (in the real world the airplane moving forward is much faster than the car moving forward).

Rightward bias. The statistical test did not support rightward bias in RM.

\section{EXPERIMENT 2}

In Experiment 1, we showed that the observers' knowledge about the objects' typical motions influenced RM. There was, however, the possibility of an alternative explanation for the results of Experiment 1. As is shown in Figure 1A, the global shape of the airplane is pointed, but that of the car is not clearly pointed. Therefore, if the pointedness of the objects affected RM, the results obtained in Experiment 1 might be explained not by a typical-motion effect, but by a pointedness effect. In Experiment 2, we examined the influence of the pointedness of objects on RM by using pictures roughly similar to the outline of the pictures used in Experiment 1. We examined rightward bias in RM, as in Experiment 1.

\section{Method}

Participants. Thirteen additional undergraduate students and one additional graduate student at Kwansei Gakuin University volunteered to participate in the present experiment. All the participants had normal or corrected-to-normal vision. They were naive as to the aim of the present experiment.

Apparatus. The apparatus was the same as that used in Experiment 1 .

Stimuli and Procedure. The stimuli and the procedure were the same as those in Experiment 1, with the exception of the pictures presented. In the present experiment, we used pictures roughly similar to the outline of the airplane and the car (Figure 1B). These outline pictures were $1.9^{\circ}$ (76 pixels) long. The outline picture of the airplane had a clear point, but that of the car did not have a clear point. According to the respective sharpness of the points, we called each outline picture P (pointed shape) or LP (less-pointed shape). In order to use the same terms as those in Experiment 1, we defined the point of the outline pictures as the front, the picture's motion in the direction of its point as forward motion, and the picture's motion in the opposite direction as backward motion. The fronts of these outline pictures corresponded to those of their original objects. The participants were not told which ends of the outline pictures were their fronts.

As in Experiment 1, there were eight conditions: the PFL, PFR, PBL, PBR, LPFL, LPFR, LPBL, and LPBR conditions. For example, in the PBR condition, the participants saw the pointed-shape picture's $(\mathrm{P})$ backward motion (in the opposite direction of its point) from the left to the center of the screen in the motion display. After 30 practice trials, each participant received 360 trials in a different random order: 2 (pictures: P or LP) $\times 2$ (moving forward or backward) $\times 2$ (moving leftward or rightward) $\times 9$ displacement of probes $\times 5$ replications. The entire session lasted about $70 \mathrm{~min}$.

\section{Results and Discussion}

The mean percentage of trials eliminated was $1.49 \%$ $(0.28 \%-2.50 \%)$. Table 2 shows the proportions of the same responses for each probe and the weighted means for each condition. The $t$ tests revealed that the weighted means for all the conditions except the PBL condition were significantly larger than zero [all $t \mathrm{~s}(13)>2.42$, all $p \mathrm{~s}<.05]$. The difference between the weighted mean for the PBL condition and zero was marginally significant $[t(13)=1.78, p=.099]$. Thus, it was confirmed that RM (forward memory shift) occurred in all the conditions except the PBL condition. In addition, RM in the PBL condition was suggested.

The weighted means were analyzed in a 2 (picture: $\mathrm{P}$ or LP) $\times 2$ (FB motion: forward or backward $) \times 2$ (LR motion: leftward or rightward) ANOVA. None of the main effects was significant. A picture $\times$ FB motion interaction $[F(1,13)=11.54, p<.01]$ and a FB motion $\times$ LR motion interaction $[F(1,13)=7.06, p<.05]$ were significant. However, the other interactions were not significant.

Pointedness effect. As in Experiment 1, the statistical concern focused on the significant picture $\times$ FB motion interaction, which reflected the larger difference in the magnitude of forward memory shift between the $P$ picture's forward (PF: $M=0.12$ ) and backward (PB: $M=$ $0.07)$ motions, but a much smaller difference between the LP picture's forward (LPF: $M=0.10$ ) and backward (LPB: $M=0.10$ ) motions. This trend is shown in Figure 3. 
Table 2

Mean Proportions of the Same Responses for Each Probe and Mean Weighted Means for Each Condition in Experiment 2

\begin{tabular}{|c|c|c|c|c|c|c|c|c|c|c|}
\hline \multirow[b]{2}{*}{ Condition } & \multicolumn{9}{|c|}{ Displacement of Probe (deg) } & \multirow[b]{2}{*}{ Weighted Mean (deg) } \\
\hline & -0.50 & -0.38 & -0.25 & -0.13 & 0 & +0.13 & +0.25 & +0.38 & +0.50 & \\
\hline \multicolumn{11}{|c|}{ Pointed pattern } \\
\hline PBL & .07 & .16 & .30 & .56 & .79 & .67 & .56 & .29 & .11 & .04 \\
\hline PBR & .06 & .10 & .19 & .53 & .67 & .74 & .66 & .44 & .19 & .10 \\
\hline \multicolumn{11}{|c|}{ Less-pointed pattern } \\
\hline LPBL & .13 & .13 & .29 & .59 & .54 & .76 & .64 & .34 & .17 & .07 \\
\hline LPBR & .04 & .07 & .20 & .33 & .80 & .73 & .69 & .56 & .23 & .13 \\
\hline
\end{tabular}

Note-The "-_" sign indicates a displacement of a probe in the opposite direction of motion of an object. The "+" sign indicates a displacement of a probe in the same direction of motion of an object. P, pointed; LP, less pointed; F, forward; B, backward; L, leftward; R, rightward.

As to the picture $\times$ FB motion interaction, post hoc Tukey's HSD tests $(p<.05)$ revealed that PF produced larger forward memory shift than did PB.

The significant interaction, similar to the result of Experiment 1 , suggested that the pointedness of the objects affected RM and implied that the results of Experiment 1 reflected the influence of pointedness. However, the post hoc tests showed the difference in results between Experiments 1 and 2. In the first experiment, the airplane's forward motion (AF) produced larger forward memory shift than did the car's forward motion (CF), but in the present experiment, there was no significant difference between the P picture's forward motion (PF) and the LP picture's forward motion (LPF). This discrepancy might suggest that, between distinct objects, there was some contribution of knowledge about the objects' typical velocities to the results of Experiment 1.

Rightward bias. Although the main effect for LR motion was not significant, the significant picture $\times$ LR motion and FB motion $\times$ LR motion interactions might sug-

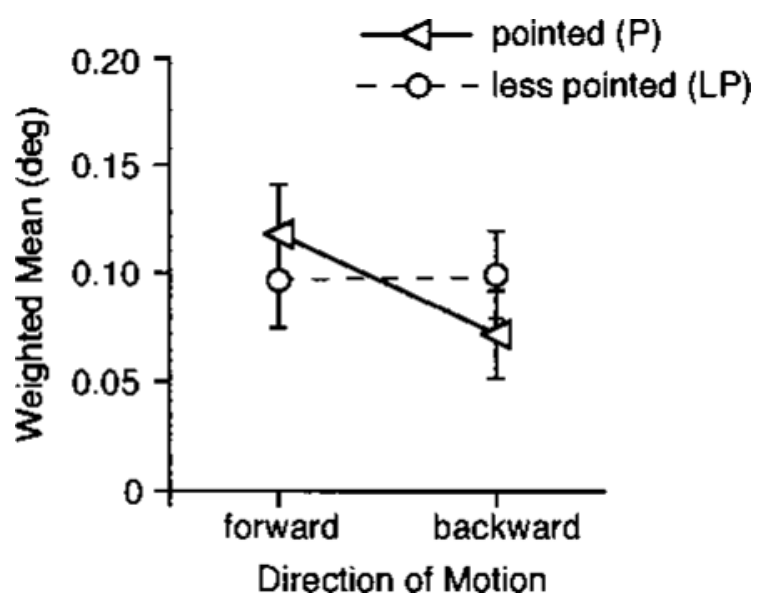

Figure 3. The weighted means for the pointed picture's forward $(P F)$ and backward (PB) motions and for the less pointed picture's forward (LPF) and backward (LPB) motions in Experiment 2. These values arose from averaging the weighted means for the conditions over the leftward/rightward motions. Error bars indicate standard errors of the mean (SEMs). gest some influences of the leftward-rightward motions on RM. The interactions would result from the objects' rightward motions' (e.g., in the PBR condition) producing larger forward memory shift than did the objects' leftward motions (e.g., the PBL condition), except for the cases in which the P picture's moving forward (the PFL and PFR conditions) did not produce such a difference (Table 2).

\section{EXPERIMENT 3}

In Experiment 2, we found an influence of the pointedness of objects by presenting the outline pictures of the objects used in Experiment 1. Thus, it was possible that the results of Experiment 1 were due to a pointedness effect, rather than to a typical-motion effect. In Experiment 1 , we did not control the shape of objects, so a typical-motion factor might be mixed with a pointedness factor. In Experiment 3, we dissociated these two factors (typical motion and pointedness) and tried to clearly control the typical directions of the objects' motions. We used pictures of four objects: a pointed (triangle-shaped) airplane and carrot and a not-pointed (cross-shaped) airplane and signpost (Figure 1C). One of the pointed objects (a triangle-shaped airplane) and one of the notpointed objects (a cross-shaped airplane) fly only forward, but the others do not move in the real world. ${ }^{3}$ In this case, it is natural to assume the influence of knowledge about the typical directions of these objects' motions, rather than that about their typical velocities, although the typical speeds of these two airplanes are somewhat different from each other.

The factorial design of the present experiment was 2 (pointedness: pointed shape or not) $\times 2$ (typical direction of motion: only forward or none) $\times 2$ (FB motion: forward or backward) $\times 2$ (LR motion: leftward or rightward). If only the pointedness factor were to influence RM, a significant pointedness $\times$ FB motion interaction would be found, as in Experiment 2. If the typical-direction factor also were to affect $\mathrm{RM}$, a significant typical direction $X$ FB motion interaction and/or a significant pointedness $X$ typical direction $\times$ FB motion interaction would be found.

As in the previous experiments, we examined rightward bias in RM. 


\section{Method}

Participants. Fourteen additional undergraduate students and one additional graduate student at Kwansei Gakuin University volunteered to participate in the present experiment. All the participants had normal or corrected-to-normal vision. They were naive as to the aim of the present experiment.

Apparatus. The apparatus was the same as in Experiment 1.

Stimuli and Procedure. The stimuli and the procedure were the same as those in Experiment 1, with the exceptions of the pictures presented and the number of replications. We used pictures of leftfacing and right-facing objects: two airplanes, a carrot, and a signpost (Figure $1 \mathrm{C}$ ). The pictures were $2.0^{\circ}$ ( 80 pixels) long. In order to use the same terms as those in Experiment 1, we defined the front of the airplanes and the point of the carrot as the front of these objects. Although the signpost seemed completely symmetrical, one side of it contained one more black pixel than did the other side of it. For the same reason and also for the purpose of data analyses, we defined the side that contained one more black pixel as the front of the signpost, although it neither moves in the real world nor has a point. The participants could not discriminate the front of the signpost. They were not told which ends of the pictures of the carrot and the signpost were their fronts. We defined the objects' motion in the direction of their fronts as forward motion and the objects' motion in the opposite direction as backward motion.

We named each object according to the combination of whether an object was expected to produce a pointedness effect $(+\mathrm{P})$ or not $(-\mathrm{P})$ and whether an object was expected to produce a typicaldirection effect $(+\mathrm{T})$ or not $(-\mathrm{T})$. Likewise, we named each condition according to the combination of an object's name and the type of motion in the motion display ( $[\mathrm{F}$ or $\mathrm{B}] \times[\mathrm{L}$ or $\mathrm{R}]$ ). There were 16 conditions: the $+\mathrm{P}+\mathrm{TFL},+\mathrm{P}+\mathrm{TFR},+\mathrm{P}+\mathrm{TBL},+\mathrm{P}+\mathrm{TBR},+\mathrm{P}-\mathrm{TFL}$, $+\mathrm{P}-\mathrm{TFR},+\mathrm{P}-\mathrm{TBL},+\mathrm{P}-\mathrm{TBR},-\mathrm{P}+\mathrm{TFL},-\mathrm{P}+\mathrm{TFR},-\mathrm{P}+\mathrm{TBL}$, $-\mathrm{P}+\mathrm{TBR},-\mathrm{P}-\mathrm{TFL},-\mathrm{P}-\mathrm{TFR},-\mathrm{P}-\mathrm{TBL}$, and $-\mathrm{P}-\mathrm{TBR}$ conditions. For example, in the $+\mathrm{P}-\mathrm{TFL}$ condition, the participants saw the carrot's $(+\mathrm{P}-\mathrm{T})$ forward motion (in the direction of its point) from the right to the center of the screen in the motion display.

After 30 practice trials, each participant received 576 trials in a different random order: 4 objects $\times 2$ (moving forward or back- ward) $\times 2$ (moving leftward or rightward) $\times 9$ displacement of probes $\times 4$ replications. The entire session lasted about $90 \mathrm{~min}$.

\section{Results and Discussion}

The mean percentage of trials eliminated was $1.79 \%$ $(0.87 \%-3.50 \%)$. Table 3 shows the proportions of the same responses for each probe and the weighted means for each condition. The $t$ tests revealed that the weighted means for all the conditions were significantly larger than zero [all $t \mathrm{~s}(14)>2.21$, all $p \mathrm{~s}<.05$ ]. Thus, it was confirmed that RM (forward memory shift) occurred in all the conditions.

The weighted means were analyzed in a 2 (pointedness: pointed or not pointed) $\times 2$ (typical direction of objects' motion: forward or none) $\times 2$ (FB motion: forward or backward) $\times 2$ (LR motion: leftward or rightward) ANOVA. It revealed that a main effect for FB motion was significant $[F(1,14)=5.25, p<.05]$ but the other three main effects were not significant. A pointedness $\times$ typical-direction interaction $[F(1,14)=8.05, p<$ $.05]$ and a pointedness $\times$ FB motion interaction $[F(1,14)=$ $10.28, p<.01]$ were significant. However, the pointedness $\times$ typical-direction $\times$ FB motion interaction and the other interactions were not significant.

Pointedness effect and typical-direction effect. As was stated in the introduction of the present experiment, the statistical concern focused on the three interactions that reflected the influences of pointedness and of the typical directions of the objects' motions. As for these two effects, Figures 4A and 4B summarize the results of Experiment 3. As in Experiment 2, the significant pointedness $\times$ FB motion interaction reflected the larger forward memory shift for the pointed objects' (the

Table 3

Mean Proportions of the Same Responses for Each Probe and Mean Weighted Means for Each Condition in Experiment 3

\begin{tabular}{|c|c|c|c|c|c|c|c|c|c|c|}
\hline \multirow[b]{2}{*}{ Condition } & \multicolumn{9}{|c|}{ Displacement of Probe (deg) } & \multirow[b]{2}{*}{ Weighted Mean (deg } \\
\hline & -0.50 & -0.38 & -0.25 & -0.13 & 0 & +0.13 & +0.25 & +0.38 & +0.50 & \\
\hline \multicolumn{11}{|c|}{ Triangle-shaped airplane } \\
\hline$+\mathrm{P}+\mathrm{TFL}$ & .03 & .15 & .24 & .38 & .60 & .63 & .61 & .45 & .32 & .13 \\
\hline$+\mathrm{P}+\mathrm{TBL}$ & .05 & .13 & .18 & .44 & .61 & .61 & .48 & .36 & .20 & .09 \\
\hline$+\mathrm{P}+\mathrm{TBR}$ & .05 & .07 & .11 & .39 & .62 & .71 & .54 & .24 & .27 & .11 \\
\hline \multicolumn{11}{|l|}{ Carrot } \\
\hline$+\mathrm{P}-\mathrm{TBL}$ & .04 & .17 & .23 & .46 & .62 & .67 & .53 & .34 & .16 & .08 \\
\hline$+\mathrm{P}-\mathrm{TBR}$ & .02 & .07 & .15 & .45 & 67 & .69 & .53 & .29 & .19 & .10 \\
\hline \multicolumn{11}{|c|}{ Cross-shaped airplane } \\
\hline$-\mathrm{P}+\mathrm{TFL}$ & .12 & .15 & .13 & .35 & .53 & .67 & .48 & .28 & .23 & .08 \\
\hline$-\mathrm{P}+\mathrm{TFR}$ & .07 & .07 & .16 & .27 & .58 & .74 & .63 & .45 & .20 & .13 \\
\hline$-\mathrm{P}+\mathrm{TBL}$ & .07 & .12 & .13 & .27 & .48 & .55 & .42 & .37 & .17 & .12 \\
\hline$-\mathrm{P}-\mathrm{TBR}$ & .00 & .00 & .11 & .27 & .66 & .63 & .58 & .33 & .11 & .13 \\
\hline
\end{tabular}

Note-The "-" sign indicates a displacement of a probe in the opposite direction of motion of an object. The "+" sign indicates a displacement of a probe in the same direction of motion of an object. $+\mathrm{P}$, pointedness; $-\mathrm{P}$, no pointedness; $+\mathrm{T}$, typical direction; $-\mathrm{T}$, no typical direction; $\mathrm{F}$, forward; B, backward; L, leftward; R, rightward. 

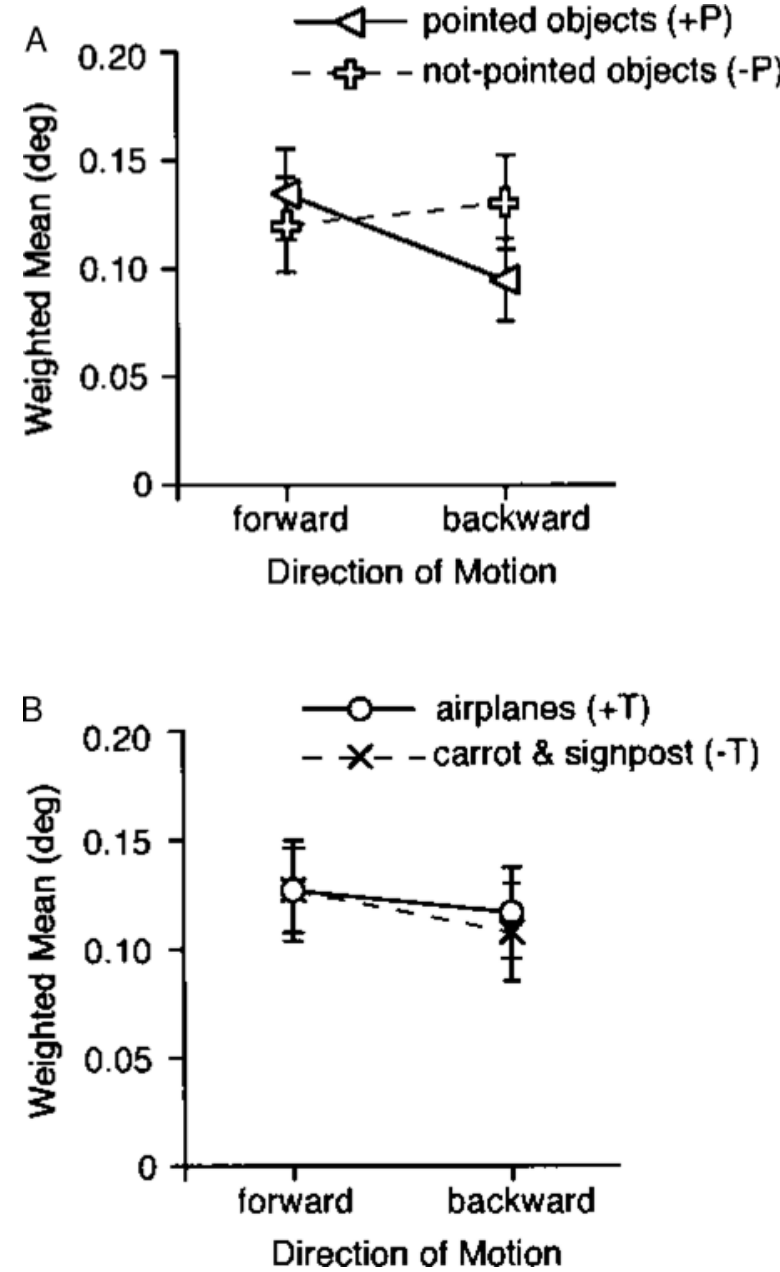

Figure 4. (A) The weighted means for the pointed objects' (the triangle-shaped airplane $[+\mathrm{P}+\mathrm{T}]$ and carrot $[+\mathrm{P}-\mathrm{T}])$ forward $(+\mathrm{PF})$ and backward $(+\mathrm{PB})$ motions and for the not-pointed objects' (the cross-shaped airplane $[-P+T]$ and signpost $[-P-T]$ ) forward $(-P F)$ and backward $(-P B)$ motions in Experiment 3. (B) The weighted means for the two airplanes' $(+P+T$ and $-P+T)$ forward (+TF) and backward (+TB) motions and for the other two objects' (the carrot $[+P-T]$ and the signpost $[-P-T]$ ) forward ( - TF) and backward (-TB) motions in Experiment 3 . The values in both panels $A$ and $B$ originated from the same data set. These values arose from averaging the weighted means for the conditions over the leftward/rightward motions and from averaging those averaged values over two objects. The latter averaging was done according to whether the objects had the point $(+P)$ or not $(-P)$ in panel $A$ or according to whether the typical direction of the objects was forward $(+T)$ or none $(-T)$ in panel $B$. Error bars indicate standard errors of the mean (SEMs).

triangle-shaped airplane $[+\mathrm{P}+\mathrm{T}]$ and carrot $[+\mathrm{P}-\mathrm{T}])$ forward motions (+PF: $M=0.13$ ) than for their backward motions (+PB: $M=0.09)$, but such a difference was not found in the not-pointed objects (the cross-shaped airplane $[-\mathrm{P}+\mathrm{T}]$ and signpost $[-\mathrm{P}-\mathrm{T}] ;-\mathrm{PF}: M=0.12$; $-\mathrm{PB}: M=0.13)$. As to the pointedness $\times \mathrm{FB}$ motion interaction, post hoc Tukey's HSD tests $(p<.05)$ revealed that $+\mathrm{PF}$ produced larger forward memory shift than did $+\mathrm{PB}$ and that $-\mathrm{PB}$ produced larger forward memory shift than did $+\mathrm{PB}$. Therefore, it was suggested that RM was influenced by the pointedness of the objects. In contrast, the insignificant typical-direction $X$ FB motion and pointedness $\times$ typical-direction $\times$ FB motion interactions suggested no influence of typical directions of objects' motions. If such an influence occurred, the weighted means for the airplanes' $(+\mathrm{P}+\mathrm{T}$ and $-\mathrm{P}+\mathrm{T}$ ) forward motions ( $+\mathrm{TF}$ ) would have been larger than their backward motions (+TB), but this difference would have been small in the other two objects (the car$\operatorname{rot}[+\mathrm{P}-\mathrm{T}]$ and the signpost $[-\mathrm{P}-\mathrm{T}])$. However, the results showed a slightly larger forward memory shift for the forward motions than for the backward motions with both the airplanes (+TF: $M=0.13 ;+\mathrm{TB}: M=0.12$ ) and the other objects ( $-\mathrm{TF}: M=0.13$; $-\mathrm{TB}: M=0.11)$. As to the cross-shaped airplane, its forward motion $(-\mathrm{P}+\mathrm{TF}$ : 0.10 ) produced smaller forward memory shift than did its backward motion ( $-\mathrm{P}+\mathrm{TB}: 0.13)$, which suggested that a typical-direction effect did not occur. The pointedness at the boundary between the cross-shaped airplane's body and the stabilizer might overcome any typicaldirection effect of it. These results suggested that RM was influenced only by pointedness, not by the typical directions of the objects' motions.

Rightward bias. Rightward bias was suggested in Experiment 2 but was not found in Experiments 1 and 3, because neither the main effect for LR motion nor the interactions related to LR motion were significant in the present experiment. Thus, the present study did not consistently show rightward bias.

Pointedness effect was weaker than momentum effect. Hubbard (1995a, 1995b, 1996) proposed that memory shift for a moving stimulus results from a combination of several factors. In the present experiments, the measured memory shift arose from the influences of the momentum and the pointedness of a moving object (we could exclude rightward bias from the possible sources, because it was not consistently found in the present study). With pointed objects, the influence of pointedness enlarged forward memory shift in the case in which the direction of the object's point and the direction of the object's motion were the same. In contrast, the influence of pointedness would diminish forward memory shift in the case in which the direction of the object's point and the direction of the object's motion were the opposite. In the latter case, even backward memory shift would be observed if the influence of pointedness was strong enough to overcame that of momentum. In the present experiments, nonetheless, the weighted means for all the pointed objects' backward motions were positive (Tables 1, 2, and 3 ). This would suggest that the pointedness effect was weaker than the momentum effect.

\section{GENERAL DISCUSSION}

The present study clearly showed that RM was influenced by the pointedness of objects, not by knowledge about the typical directions of objects' motions, and showed that the pointedness effect was weaker than the 
momentum effect. As was stated in the results section of Experiment 2, the discrepancy in results between Experiments 1 and 2 suggested some influence of a typicalvelocity factor. However, there was a possibility that this factor was not completely controlled, because we employed only one speed for the objects' motions in the motion display, regardless of the objects (the airplanes, the car, etc.). Therefore, we should not conclude that a typicalvelocity effect occurred. Rightward bias was not consistently found in the present three experiments. Further studies would be required to conclude whether rightward bias is observed in RM.

A pointedness effect was also found in an apparent motion study (McBeath, Morikawa, \& Kaiser, 1992) and in a study of memory distortion by static stimuli (Freyd $\&$ Pantzer, 1995). These findings suggested that the pointedness of objects influences the perceptual system and, hence, RM. As to the typical-motion effect (the typical-direction effect and the typical-velocity effect), the results of several studies vary. Although Reed and Vinson (1996) showed a typical-direction effect, we did not. Halpern and Kelly (1993) did not find a typicalvelocity effect. In the apparent motion study, a typicaldirection effect was found, but its influence was weaker than the pointedness effect (McBeath et al., 1992). These findings might imply that knowledge about object-specific motions has a weaker influence on RM than does the pointedness of objects.

Consistent with Shepard's $(1984,1994)$ speculation that human cognitive activities are guided by the internalization of long-enduring physical laws, Freyd and her collaborators suggested that RM occurs because the law of physical momentum has been internalized in the human representational system (Finke \& Freyd, 1985; Kelly \& Freyd, 1987). Along these lines, Hubbard (1995b) explicitly suggested that not only momentum, but also other physical principles (e.g., gravitational attraction and frictional drag) are invariant over the course of human evolution and, thus, might have been incorporated into the representational system. As to the relative strengths of the influences of principles in the real world, Shepard (1984) made a proposal that the automatic operations of the perceptual processes should be guided more by general principles than by the specific behaviors of objects. Whether a principle is general or specific would depend on the frequency of the experiences that people are exposed to. All objects in motion possess momentum, irrespective of their shapes, the typical directions of their motions, and so on. In consequence, people experience the physical momentum principle frequently. As for the pointedness effect, a pointed object receives weaker frictional drag when moving in the direction of its point than when moving in the opposite direction. This relationship between pointedness and friction is general but somewhat specific, because the pointedness depends on the shapes of objects (some objects have points, but others do not). Accordingly, people experience the pointedness principle less frequently or less strongly than they do the law of momentum. Therefore, the momentum principle would have been incorporated more deeply into human representational system and would influence RM more strongly than would the pointedness principle.

RM occurred both with apparent/smooth motion (e.g., Hubbard, 1990, 1995a) and with implied/discrete motion (e.g., Freyd \& Finke, 1984; Reed \& Vinson, 1996). Faust (1990) showed that RM arose irrespective of the quality of motion. However, we should notice that the quality of motion is important for the interpretation of the present results, because the locus of the activation in the cortex depends on the quality of motion. It has been suggested that apparent motion activates visual motion detectors in the striate (V1) and middle temporal (MT) areas, whereas implied motion does not (Mikami, Newsome, \& Wurtz, 1986). In the present experiments, therefore, only local features (pointedness or oblique lines), which are supposed to be processed in the striate or the temporal areas, might strongly effect RM. Consequently, the pointedness factor might overshadow such a cognitive factor as knowledge about objects' typical motions. In contrast, implied motion might affect a solely cognitive mechanism and might produce a typical-direction effect (Reed \& Vinson, 1996). Nevertheless, an expectation effect, which could be considered as a kind of $\operatorname{cog}$ nitive effect on RM, was found with both implied motion (Verfaillie \& d'Ydewalle, 1991) and smooth motion (Hubbard \& Bharucha, 1988). A typical-direction effect would result from knowledge about objects' typical directions of motions, which are stored in long-term memory. That knowledge would be automatically activated by the identification of objects. On the other hand, an expectation effect would originate from the knowledge or expectation caused by watching the periodic motion of an object. This knowledge would not be activated automatically. We can point out the possibility that the different sources of these cognitive effects might lead to the discrepancy in results. However, it is impossible to give clear explanations from the present results.

As to stimulus selection, it is very difficult to control both pointedness and typical-direction factors when the stimuli resemble or depict familiar three-dimensional physical objects. Although we controlled typical directions of objects' motions in Experiment 3, we could not equalize the pointedness of objects perfectly, because we gave priority to the graphic depiction of the stimuli. On the other hand, Reed and Vinson (1996) controlled both pointedness and typical-direction factors, but whether or not the stimuli resembled actual objects was of secondary importance in their study. The present result, that the stimuli depicted more graphically did not produce a typical-direction effect, suggests the importance of quality of motion in interpreting the present results.

\section{REFERENCES}

FAust, M. (1990). Representational momentum: A dual process perspective. Unpublished doctoral dissertation, University of Oregon, Eugene. 
Finke, R. A., \& Freyd, J. J. (1985). Transformations of visual memory induced by implied motions of pattern elements. Journal of Experimental Psychology: Learning, Memory, \& Cognition, 11, 780-794.

Finke, R. A., Freyd, J. J., \& Shy , G. C. W. (1986). Implied velocity and acceleration induce transformations of visual memory. Journal of Experimental Psychology: General, 115, 175-188.

Freyd, J. J., \& FinKe, R. A. (1984). Representational momentum. Journal of Experimental Psychology: Learning, Memory, \& Cognition, 10, 126-132.

Freyd, J. J., \& Finke, R. A. (1985). A velocity effect for representational momentum. Bulletin of the Psychonomic Society, 23, 443-446.

Freyd, J. J., \& Miller, G. F. (1992, November). Creature motion. Paper presented at the 33rd Annual Meeting of the Psychonomic Society, St. Louis.

Freyd, J. J., \& PAntzer, T. M. (1995). Static patterns moving in the mind. In S. M. Smith, T. B. Ward, \& R. A. Finke (Eds.), The creative cognition approach (pp. 181-204). Cambridge, MA: MIT Press.

HalPern, A. R, \& Kelly, M. H. (1993). Memory biases in left versus right implied motion. Journal of Experimental Psychology: Learning, Memory, \& Cognition, 19, 471-484.

Hubbard, T. L. (1990). Cognitive representation of linear motion: Possible direction and gravity effects in judged displacement. Memory \& Cognition, 18, 299-309.

HubBard, T. L. (1995a). Cognitive representation of motion: Evidence for representational friction and gravity analogues. Journal of Experimental Psychology: Learning, Memory, \& Cognition, 21, 241 254

HubBard, T. L. (1995b). Environmental invariants in the representation of motion: Implied dynamics and representational momentum, gravity, friction, and centripetal force. Psychonomic Bulletin \& Review, 2, 322-338

Hubbard, T. L. (1996). Representational momentum, centripetal force, and curvilinear impetus. Journal of Experimental Psychology: Learning, Memory, \& Cognition, 22, 1049-1060.

Hubbard, T. L. (1998). Representational momentum and other displacements in memory as evidence for nonconscious knowledge of physical principles. In S. R. Hameroff, A. W. Kaszniak, \& A. C. Scott (Eds.), Towards a science of consciousness: II. The second Tucson discussions and debates (pp. 505-512). Cambridge, MA: MIT Press.

HubB ARD, T. L. (1999). How consequence of physical principles influence mental representation: The environmental invariants hypothesis. In P. R. Killeen \& W. R. Uttal (Eds.), Fechner day 99: The end of 20th century psychophysics. Proceedings of the 15th Annual Meeting of the International Society for Psychophysics (pp. 274-279). Tempe, AZ: International Society for Psychophysics.

Hubbard, T. L., \& Bharucha, J. J. (1988). Judged displacement in apparent vertical and horizontal motion. Perception \& Psychophysics, 44, 211-221.

Kelly, M. H., \& Freyd, J. J. (1987). Extrapolations of representational momentum. Cognitive Psychology, 19, 369-401.

McBeath, M. K., Morikawa, K., \& Kaiser, M. K. (1992). Perceptual bias for forward-facing motion. Psychological Science, 3, 362-367.
Mikami, A., Newsome, W. T., \& Wurtz, R. H. (1986). Motion selectivity in macaque visual cortex: II. Spatiotemporal range of directional interactions in MT and V1. Journal of Neurophysiology, 55, $1328-1339$

ReEd, C. L., \& Vinson, N. G. (1996). Conceptual effects on representational momentum. Journal of Experimental Psychology: Human Perception \& Performance, 22, 839-850.

SHEPARD, R. N. (1984). Ecological constraints on internal representation: Resonant kinematics of perceiving, imaging, thinking, and dreaming. Psychological Review, 94, 417-447.

ShePARD, R. N. (1994). Perceptual-cognitive universals as reflections of the world. Psychonomic Bulletin \& Review, 1, 2-28.

Verfaillie, K., \& D’Ydewalle, G. (1991). Representational momentum and event course anticipation in the perception of implied periodical motions. Journal of Experimental Psychology: Learning, Memory, \& Cognition, 17, 302-313.

\section{NOTES}

1. On the ground, an airplane can move forward and backward with its own self-generated power or can be moved forward and backward by such as a trailer. However, the airplane moves on the ground only just before it takes off and just after it lands. The airplane moves forward almost all the time when it flies. Therefore, we did not consider the airplane's motion on the ground as its typical motion and assumed that the airplane's typical velocity in its backward motion is zero.

2. In order to discriminate among the conditions, the participants were to identify an object and the front of that object in the motion display. We investigated the accuracy of identification of all 16 pictures used in this study ( 8 objects $\times$ left/right-facing one) by presenting those pictures in motion to an additional group of 10 observers who did not participate in the RM task. They saw the motion display in which one of 16 pictures moved leftward or rightward and were asked to select the picture presented in the motion display from 16 pictures presented subsequently. For objects that have no front, we defined the point of the carrot and one side of the signpost as their front, in order to use the same term in the present study. The observers were not told which end of the pictures was their fronts. In order to perform this task, they should identify the object and its front in the motion display. The result showed that all the pictures were perfectly identified, except for the signposts used in Experiment 3 (near-chance performance).

3. As in Experiment 1, we considered that a typical direction of the airplanes' motion was only forward. The carrot and the signpost cannot move with their own self-generated power, although they can be moved by human or other forces. In addition, people rarely see motion of the carrot or the signpost. Thus, we did not consider these passive motions as their typical motions.

(Manuscript received July 13, 1998; revision accepted for publication May 30, 2000.) 